\title{
NUEVAS TERAPIAS EN DIABETES: MÁS ALLÁ DE LA INSULINA INYECTABLE Y DE LOS ANTIDIABÉTICOS ORALES
}

\author{
John Edwin Feliciano Alfonso*, Iván Darío Sierra Ariza \\ Trabajo realizado por División de Lípidos y Diabetes Facultad de Medicina Universidad Nacional de Colombia, Bogotá, Colombia
}

\author{
*Correspondência: \\ Laboratorio 418. División \\ de Lípidos y Diabetes. \\ Facultad de Medicina, \\ Ed 471. Ciudad \\ Universitaria Carrera 30 No \\ 45-03. \\ Universidad Nacional de \\ Colombia, Bogotá D.C., \\ Colombia. \\ Teléfono: 3165000 , \\ Ext: $15054 ; 3165479$ \\ jefelicianoa@yahoo.com
}

\begin{abstract}
RESUMO
Novos medicamentos para o tratamento do diabetes tipo I e tipo 2 foram incorporados à lista de fármacos tradicionais: antidiabéticos orais e insulinas injetáveis. Estas alternativas de tratamento têm novos mecanismos de ação que aproveitam as propriedades antidiabéticas de certos peptídeos como é o caso da amilina ou do peptídeo similar ao glucagon (GLP-I), cujos níveis são deficientes ou insuficientes no diabetes. Isto acontece pelos análogos da amilina ou do GLP-I, embora também possa ser obtido inibindo a enzima que degrada este último. Além disso, encontra-se disponível no mercado um novo sistema para administrar insulina de maneira não-invasiva por meio de inalação. Este artigo resume os resultados mais importantes e atualizados com relação ao mecanismo de ação, eficácia, efeitos adversos e indicações destes fármacos inovadores.
\end{abstract}

Unitermos: Agentes hipoglucémicos. Diabetes Mellitus tipo I. Diabetes Mellitus tipo 2. Péptido I similar ao glucagon. Dipeptidil peptidasa IV. Insulina.

\section{INTRODUCCIÓN}

Hasta hace poco tiempo la terapia para la diabetes mellitus se circunscribía a seis clases de familias farmacológicas: las sulfonilureas, las meglitinidas, las biguanidas, las tiazolidindionas, los inhibidores de las alfa glucosidasas intestinales y las insulinas. Sin embargo, medicamentos con mecanismos de acción novedosos son el resultado de la investigación cientifica en diabetes recientemente. De esta manera, las opciones de tratamiento farmacológico se han ampliado actualmente, abriendo paso a tres nuevas familias de medicamentos antidiabéticos: los agonistas del péptido I similar al glucagón (GLP-I), los inhibidores de la dipeptidil peptidasa IV (DPP-IV) y los agonistas de la amilina. De igual forma, ya se encuentraba disponible una presentación de insulina para ser administrada por inhalación.

El buen control de la diabetes mellitus es una prioridad en los países latinoamericanos, ya que se le reconoce como un problema de salud pública serio, costoso, frecuente y creciente!. Por esta razón, el conocimiento de las nuevas alternativas farmacológicas se hace necesario para poder realizar una correcta prescripción en los pacientes apropiados.

A continuación, se revisará de forma práctica cada una de estas familias de medicamentos, haciendo énfasis en tres elementos claves: mecanismo de acción, efectos adversos y evidencia que avala su uso en diabetes mellitus.

\section{Agonistas Glp- I}

\section{Mecanismo de acción}

El GLP-I es uno de los péptidos intestinales que potencian la secreción de insulina en respuesta a la ingestión de nutrientes. Esta propiedad se conoce con el nombre de «efecto incretina»². Es liberado en las células «L» enteroendocrinas del intestino delgado distal y del colon específicamente cuando la glucemia está elevada, y su vida media es muy corta $(<2 \mathrm{~min})$, debido a que es rápidamente inactivada por la enzima proteolítica DPP-IV³. Las múltiples acciones del GLP-I, que se resumen a continuación, se producen cuando se une a su receptor acoplado a proteína $G(G L P-\mid R)$, el cual está localizado en varios tejidos ${ }^{4}$ :

- Páncreas: Aumento de la secreción de insulina tras la ingestión de alimentos, inhibición de la secreción de glucagón por las células a $a^{5}$, aumento de la proliferación y prevención de apoptosis de las células b (esta última propiedad demostrada en animales de experimentación o en islotes humanos in vitro) ${ }^{6,7}$.

- Estómago: Disminución del vaciamiento gástrico8.

- Cerebro: Disminución del apetito?.

Todas estas acciones pueden ser aprovechadas para tratar a los pacientes con diabetes mellitus tipo 2 (DM2), ya que poseen ciertos defectos que pueden ser corregidos con la terapia incretina a través de los agonistas del GLP-I (Figura I).

Existen dos análogos del GLP-I aprobados para el tratamiento de la DM2: exenatide y liraglutide, resistentes a la inactivación por DPP-IV, que comparten el mecanismo de acción al imitar los efectos del GLPI, tras su unión a su receptor".

\section{Exenatide: evidencia}

Exenatide es un péptido sintético estructuralmente idéntico a la exendina 4, un péptido natural aislado de la saliva de un lagarto (monstruo de Gila) ${ }^{10}$, resistente a la inactivación por DPP-IV. Exenatide se ha evaluado en varios ensayos clínicos aleatorizados y controlados con placebo. Se incluyeron pacientes con DM2, que no lograban un 


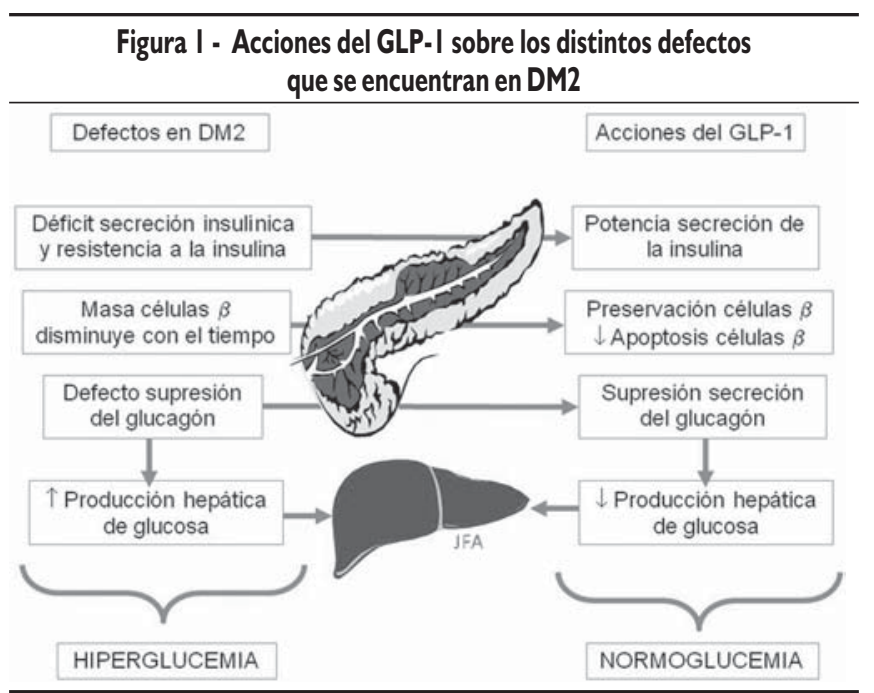

Adicionalmente el GLP-I puede retrasar el vaciamiento gástrico y disminuir el apetito, factores que no solamente colaboran con la disminución de la glucemia, sino que también permiten un descenso en el peso.

buen control a pesar de estar siendo tratados con sulfonilureas ${ }^{\prime \prime}$, metformina ${ }^{12}$ o su combinación ${ }^{13}$ y se aleatorizaron a placebo, exenatide 5 ó $10 \mathrm{mcg}$. Al final de las 30 semanas de estudio la reducción promedio de hemoglobina glucosilada $\mathrm{Al} c(\mathrm{HbAlc})$, para el grupo de exenatide 10 mcg en el análisis por intención a tratar (intention to treat) fue de:

- Exenatide $10 \mathrm{mcg}+$ sulfonilurea $={ }^{-} 0.96 \% \mathrm{HbAlc}$

- Exenatide $10 \mathrm{mcg}+$ metformina $={ }^{-} 0.90 \% \mathrm{HbAlc}$

- Exenatide $10 \mathrm{mcg}+$ sulfonilurea/metformina $={ }^{-} 1.00 \% \mathrm{HbAlc}$

De igual manera, se evidenció que más pacientes lograban una HbAlc $£ 7 \%$ en el grupo de exenatide 10 mcg y que se lograba una disminución progresiva en el peso, alrededor de I.5 kilos, con mayores reducciones si se emplea en combinación con metformina ${ }^{12}$.

Otro estudio clínico aleatorizado y controlado con placebo evaluó la adición de exenatide $10 \mathrm{mcg}$ en pacientes que no tenían un buen control con tiazolidindionas, con o sin metformina, y a pesar del corto seguimiento ( 16 semanas) se evidenció una reducción de HbAlc en $0.98 \%$ y una disminución en el peso de 1.5 kilos $^{14}$.

Otro estudio comparó la adición de exenatide contra insulina glargina ${ }^{15}$ en pacientes con DM2, que no tenían un control adecuado con antidiabéticos orales (sulfonilurea + metformina) y se evidenció que aunque se lograba una reducción de HbAl c similar, la terapia con exenatide producía una reducción en el peso de 2.3 kilos, mientras que la terapia con insulina aumentaba el peso corporal en 1.8 kilos al final de las 26 semanas de seguimiento. Otro estudio que evaluó la adición de exenatide o insulina aspartato bifásica, en el mismo perfil de pacientes, tuvo resultados similares a un año de seguimiento ${ }^{16}$.

\section{Exenatide: efectos adversos}

Por potenciar la secreción de insulina, la hipoglucemia es un efecto adverso a considerar. Sin embargo, la hipoglucemia es infrecuente y ocurre principalmente cuando se combina con sulfonilureas. Otros eventos adversos más frecuentes son la náusea, que se presenta en alrededor de la mitad de los pacientes, vómito y diarrea (aprox. 10\%). Aunque se han detectado anticuerpos contra exenatide en $40 \%$ de los pacientes, no parecen revestir importancia clínical $^{10-13}$.

\section{Exenatide: presentación e indicaciones}

Exenatide (Byetta $囚$ ) viene en lápices dispensadores prellenados que poseen $5 \mathrm{mcg}$ y $10 \mathrm{mcg}$ con 60 dosis del medicamento para cubrir el mes de tratamiento. Por ser un péptido debe ser inyectado por vía subcutánea en el muslo, brazos o abdomen, y por tener una vida media corta de una hora a hora y media ${ }^{17}$, debe ser aplicado dos veces al día: una hora antes del desayuno y de la cena. Se comienza con una dosis de $5 \mathrm{mcg}$ dos veces al día y, de acuerdo a la respuesta obtenida luego del primer mes de tratamiento, puede aumentarse a 10 mcg dos veces al día ${ }^{18}$.

Está indicado en pacientes que no alcanzan un buen control glucémico, a pesar de ser tratados en monoterapia con sulfonilurea, metformina o tiazolidindionas, o con las combinaciones de sulfonilurea + metformina o tiazolidindionas + metformina, y se debe considerar disminuir la dosis de sulfonilurea si se adiciona exenatide para disminuir la probabilidad de que se presenten hipoglucemias ${ }^{19}$. Debe aclararse que no debe ser utilizado en pacientes con diabetes mellitus tipo I o en aquellos con complicaciones agudas de la diabetes ${ }^{18}$. También se contraindica en enfermedad gastrointestinal severa e insuficiencia renal estadio IV.

\section{Exenatide: otras consideraciones}

Una presentación de exenatide de liberación prolongada (exenatide LAR: Long Acting Release) permite una administración parenteral una vez a la semana al encapsular el péptido en microesferas poliméricas ${ }^{4}$ que son lentamente degradadas, liberando la sustancia en forma controlada. En estudios de fase 2, exenatide LAR ( $2.0 \mathrm{mg}$ una vez a la semana) ha demostrado disminuir la HbAlc en $2.1 \%$ comparado con placebo en pacientes con DM2 sin buen control, a pesar de la terapia con metformina y/o dieta + ejercicio ${ }^{20}$.

\section{Liraglutide: evidencia}

Es un péptido de diseño en el cual se le hacen dos modificaciones al esqueleto de aminoácidos del GLP- I : en la posición 34 se adiciona arginina y se suprime lisina, y en la posición 26 se añade ácido glutámico más ácido palmítico, lo que le permite unirse a la albúmina y resistir la degradación por DPP-IV²1. Liraglutide ha sido evaluado en un ensayo clínico aleatorizado y controlado con placebo, donde se incluyó a pacientes con DM2 que no tenían un buen control metabólico y comparó Liraglutide a distintas dosis contra placebo ${ }^{22}$. Al final de las 14 semanas de estudio, la reducción promedio de HbAlc para el grupo de mayor dosis de liraglutide (1.90 mg) comparada con placebo fue de $1.74 \%$.

De igual manera, se evidenció que más pacientes lograban una $\mathrm{HbAl}$ c $€ 7 \%$ en el grupo de liraglutide $1.90 \mathrm{mg}$ y que se lograba una disminución en el peso de 1.2 kilos $^{22}$.

Otro estudio demostró que liraglutide a dosis de $0.75 \mathrm{mg}$ podía disminuir la $\mathrm{HbAlc}$ en una misma proporción que la ofrecida por glimepirida en $0.75 \%{ }^{23}$, y otra investigación halló que la eficacia de 
liraglutide a esta dosis en disminuir la HbAlc es comparable a metformina $500 \mathrm{mg}$ dos veces al día ${ }^{24}$.

Liraglutide fue evaluado en estudios de fase 3 (Programa LEAD: Liraglutide Effect and Action in Diabetes), cuyo objetivo es establecer la eficacia y seguridad de liraglutide (en combinación con sulfonilurea o metformina) en más de 2000 pacientes con DM2, inadecuadamente controlados con antidiabéticos orales, y cuyos resultados esperan ser publicados en el año $2008^{25}$. Análisis preliminares de algunos estudios del programa LEAD han demostrado que en el grupo de liraglutide más pacientes alcanzan una $\mathrm{HbAlc}<7 \%$ y pierden más peso respecto a los controles ${ }^{25}$.

\section{Liraglutide: efectos adversos}

De acuerdo con la evidencia disponible, los casos de hipoglucemia con liraglutide son inusuales, pues no se reportaron episodios de hipoglucemia en el estudio contra placebo ${ }^{22}$. En el estudio que comparó liraglutide con glimepirida hubo menos casos de hipoglucemia con el análogo GLP-I (6\%), que con la sulfonilurea (35\%)23,26.

Otros efectos adversos son de carácter intestinal (alrededor del $35 \%)$, entre los que se destacan la diarrea ( $(20 \%$ ) y la náusea (»10\%) No se han detectado anticuerpos contra liraglutide ${ }^{22-24}$.

Debe ser aplicado vía subcutánea una vez al día, debido a que su vida media es de aproximadamente 10 horas ${ }^{27}$.

Se debe resaltar, que los análogos del GLP-I nunca deben ser considerados como un sustituto de la insulina, y no deben prescribirse en aquellos pacientes con indicación perentoria de insulina.

\section{INHIBIDORES DPP-IV}

\section{Mecanismo de acción}

Otra oportunidad para aprovechar las propiedades antidiabéticas del GLP-I es evitar que sea inactivada tan rápidamente por la DPP-IV. Basados en esta idea se han desarrollado fármacos que inhiben la actividad de esta enzima, entre ellos encontramos: sitagliptina y vildagliptina. La terapia con inhibidores DPP-IV puede elevar los niveles plasmáticos de GLP- ${ }^{28,29}$, permitiendo que se acople a su receptor para producir todos los efectos referidos previamente. Es de resaltar, que los inhibidores de la DPP-IV no tienen mayores efectos en el retraso del vaciamiento gástrico, ni en la disminución del peso (Figura 2).

\section{Sitagliptina: evidencia}

Fue el primer inhibidor de la DPP-IV aprobado para hacer parte del arsenal terapéutico en DM2. La eficacia de sitagliptina se evaluó en un reciente meta análisis ${ }^{26}$, que incluyó estudios clínicos aleatorizados controlados con placebo, con sitagliptina como monoterapia o adicionándolo a la terapia con metformina o pioglitazona, en pacientes con DM2 sin buen control. Los estudios tuvieron una duración entre 3 y 6 meses. En general, sitagliptina puede reducir la $\mathrm{HbAl}$ c en promedio $0.74 \%$, sin cambios clínicamente significativos en el peso. Adicionalmente, se pudo establecer que más pacientes con sitagliptina alcanzaban una $\mathrm{HbAlc}<7 \%$.

Un estudio clínico aleatorizado con doble enmascaramiento evaluó la adición de sitagliptina contra una sulfonilurea en más de 1000 pacientes con DM2, inadecuadamente controlados con metformina en monoterapia ${ }^{30}$. Al final del año de seguimiento se determinó que ambas

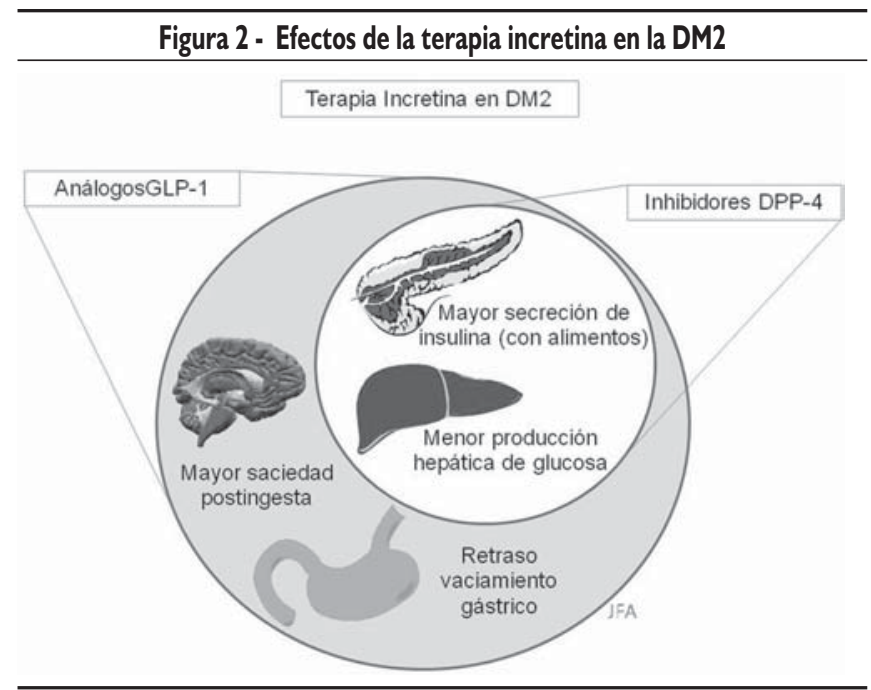

El efecto neto de los inhibidores de DPP-4 es reducir la glucosa. Los agonistas de GLP-I tienen efecto adicional sobre el peso.

alternativas tenían una eficacia comparable, con un perfil más favorable en el peso corporal en el grupo de sitagliptina.

Otro estudio clínico aleatorizado, con doble enmascaramiento controlado con placebo evaluó la eficacia de la combinación metformina + sitagliptina en más de 1000 pacientes con DM2, inadecuadamente controlados con dieta y ejercicio ${ }^{31}$. Al final de los 6 meses de seguimiento se evidenció que la combinación metformina 2000 mg + sitagliptina 100 mg (divididas en dos dosis al día), lograba una reducción de la HbAl c de $2.07 \%$ y más de la mitad de los pacientes alcanzaban una $\mathrm{HbAlc}<7 \%$.

\section{Sitagliptina: efectos adversos}

Por su mecanismo de acción hay que tener presente la hipoglucemia como efecto adverso. Sin embargo, los estudios clínicos controlados han demostrado que la incidencia de hipoglucemia es similar a placebo ${ }^{26}$. Esto puede explicarse por el hecho de que el GLP-I sólo potencia la secreción de insulina en presencia de alimento. Debido a que DPP-IV inactiva otros péptidos con alanina o prolina en la segunda posición de su extremo NH2 terminal, puede interferir con la actividad de varias citoquinas o de la sustancia P, lo que puede llevar a reacciones inmunes o inflamatorias. Sin embargo, la interferencia con otros péptidos sólo se observó in Vitro, y estos efectos no han sido reportados en humanos ${ }^{3}$. De hecho, es un fármaco bien tolerado. Sus efectos adversos gastrointestinales (náusea, diarrea, dolor abdominal) son comparables al grupo control. Pero con sitagliptina, los cuadros de nasofaringitis (6.4\%) o de infección de vías urinarias (3.2\%) son más comunes que con placebo ${ }^{26}$.

\section{Sitagliptina: presentación e indicaciones}

Sitagliptina (Januvia $®$ ) viene en tabletas de $100 \mathrm{mg}$ para ser ingerido una vez al día en pacientes con DM2, inadecuadamente controlados con dieta y ejercicio o en combinación con metformina o tiazolidindionas, cuando con estos agentes no se logre un buen contro ${ }^{32}$. 
No debe ser utilizado en pacientes con diabetes mellitus tipo I o en aquellos con DM2 inestables ${ }^{18}$. En casos de insuficiencia renal crónica estadio III debe reducirse la dosis a la mitad $(50 \mathrm{mg})$, y debe ser suspendida si la enfermedad renal progresa.

\section{Sitagliptina: otras consideraciones}

También se encuentra disponible una presentación que combina sitagliptina (50 mg) con metformina (500 mg - 1000 mg) en una misma tableta, para ser administrada dos veces al día con las comidas ${ }^{33}$. En este contexto deben contemplarse todas las observaciones de tolerancia y seguridad con metformina.

\section{Vildagliptina: evidencia}

Es el más reciente inhibidor de la DPP-IV aprobado para el tratamiento farmacológico de la DM2. La eficacia de vildagliptina se evaluó en un reciente meta-análisis ${ }^{26}$ que incluyó estudios clínicos aleatorizados controlados con placebo, con vildagliptina como monoterapia o adicionándolo a la terapia con metformina, pioglitazona o insulina en pacientes con DM2 sin buen control. Los estudios tuvieron una duración entre 3 y 6 meses. En general, vildagliptina puede reducir la $\mathrm{HbAlc}$ en promedio $0.73 \%$, sin cambios clínicamente significativos en el peso. Adicionalmente, se pudo establecer que más pacientes con vildagliptina alcanzaban una $\mathrm{HbAlc}<7 \%$.

Dos estudios clínicos aleatorizados compararon la eficacia de vildagliptina contra pioglitazon ${ }^{34}$ y rosiglitazona $a^{35}$, en más de 300 y 600 pacientes con DM2 inadecuadamente controlados con metformina en monoterapia ${ }^{30}$. Al final de los seis meses de seguimiento se determinó que ambas alternativas tenían una eficacia comparable con un perfil más favorable en el peso corporal en el grupo de vildagliptina. Sin embargo, cuando se comparó con metformina resultó menos eficaz, disminuyendo la HbAlc en $1.0 \%$ contra - $1.4 \%$ de la biguanida ${ }^{36}$.

\section{Vildagliptina: efectos adversos}

Los estudios clínicos controlados han demostrado que la incidencia de hipoglucemia es similar al placebo ${ }^{26}$. Como se anotó previamente, al inhibir la DPP-IV se podría alterar el clivaje de otros péptidos diferentes al GLP- I y que están relacionados con la función inmune. De hecho, varias enfermedades se han asociado con alteraciones en la actividad de la DPP-IV como la artritis reumatoide, la esclerosis múltiple o el lupus eritematoso sistémico ${ }^{37}$, pero no se han reportado eventos adversos de este tipo en humanos ${ }^{3}$. Al igual que sitagliptina, es un fármaco bien tolerado. Sus efectos adversos gastrointestinales (náusea, diarrea, dolor abdominal) son comparables al grupo control. Pero con vildagliptina los cuadros de infección de vías urinarias (3.2\%) o de cefalea (5. $1 \%$ ) son más comunes que con placebo ${ }^{26}$.

\section{Vildagliptina: presentación e indicaciones}

Vildagliptina (Galvus ${ }^{\circledR}$ ) se presenta en comprimidos de 50 y 100 mg para ser administrada una vez al día en la mañana, aunque puede darse en dos tomas separadas de $50 \mathrm{mg}$ dos veces al día. En monoterapia y en combinación con metformina o con tiazolidindiona se recomienda una dosis de 50 ó 100 mg, pero en combinación con sulfonilurea la dosis recomendada es de $50 \mathrm{mg}$. Se indica en pacientes con DM2, inadecuadamente controlados con dieta y ejercicio o en combinación con metformina, tiazolidindionas, sulfonilureas, o insulina cuando con éstos agentes no se alcance un buen contro ${ }^{38}$.

\section{Agonistas de la AMilina \\ Mecanismo de acción}

La amilina es también llamada polipéptido amiloide del islote. Se almacena y se cosecreta junto con la insulina en las células beta pancreáticas y su receptor pertenece a la familia del receptor de calcitonina y adrenomodulina ${ }^{39}$. Su liberación es estimulada por la ingestión de alimentos, glucagón, GLP-I y agonistas colinérgicos, mientras que es inhibida por la somatostanina y la insulina ${ }^{40}$. Sus acciones son similares a las del GLP-I, con la diferencia que no potencia la secreción de insulina.

El pramlintide es el primer análogo sintético de la amilina, desarrollado para impedir la tendencia que tiene la amilina humana de agregarse, formar partículas insolubles y adherirse a las superficies $^{41}$. Los receptores de amilina, y por ende, de pramlintide se encuentran especialmente en el sistema nervioso central, donde regula varios tejidos periféricos como el páncreas (inhibiendo la secreción de glucagón postprandial, y por tanto evitando la producción hepática de glucosa) y el tracto gastrointestinal (retardando el vaciamiento gástrico) ${ }^{41-43}$. Adicionalmente, también tiene efectos centrales importantes como reducir la ingesta de alimentos, promoviendo de esta manera la saciedad $^{44,45}$ (Figura 3). Estos efectos son de interés para tratar la diabetes, aunque se ha demostrado que los niveles de amilina son deficientes en $\mathrm{DMI}^{46}$ e insuficientes en estado postprandial en $\mathrm{DM} 2^{47}$.

\section{Pramlintide: evidencia}

El pramlintide fue evaluado en dos ensayos clínicos aleatorizados y controlados con placebo. Entre ambos estudios se incluyeron más de mil pacientes con DM2, que venían en tratamiento con insulina en monoterapia o en combinación con antidiabéticos orales (metformina o sulfonilúrea) $)^{48,49}$. Al final del año de seguimiento, la reducción promedio de $\mathrm{HbAl}$ c fue de $0.6 \%$ en ambos estudios con la máxima dosis empleada de pramlintide ${ }^{48,49}$. De igual manera, se obtuvo una reducción de peso de alrededor de 2.2 kilos respecto a placebo ${ }^{48,49}$. Existe evidencia de que la eficacia de pramlintide en reducción de $\mathrm{HbAlc}$ y peso puede ser menor en la raza hispana ${ }^{50}$.

Este medicamento también ha sido evaluado en pacientes con DMI en varios ensayos clínicos controlados aleatorizados, demostrando que la adición de diferentes dosis de pramlintide al régimen de insulina puede reducir la $\mathrm{HbAlc}$ de forma modesta (alrededor de $0.4 \%)^{51,52}$, evitando la ganancia de peso característica de la terapia con insulina, e incluso llegando a perder entre 0.3 y 1.8 kilos $^{52}$.

\section{Pramlintide: efectos adversos}

Se describen predominantemente síntomas de origen gastrointestinal: náusea en I de cada 4 pacientes con DM250, generalmente al inicio del tratamiento y de intensidad leve o moderada ${ }^{48,49}$. La frecuencia de náusea es mayor en pacientes con 


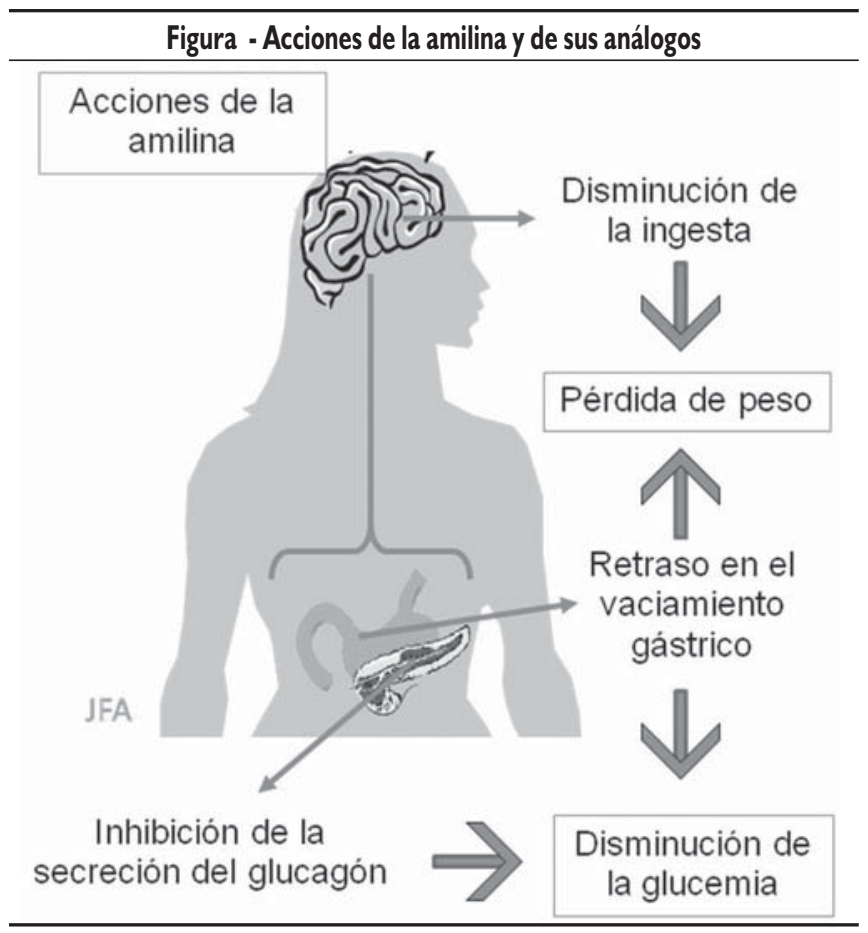

Disminución de peso y reducción de la glucemia. Ésta última se logra sin potenciar la acción de la insulina.

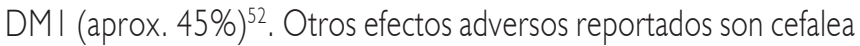
y vómito (aprox. 10\% ${ }^{49,52}$. Se debe tener en cuenta que pramlintide por sí solo no causa hipoglucemia, ya que no potencia la secreción de insulina. Sin embargo, como su uso se indica junto con insulinoterapia, debe reducirse a la mitad las dosis de insulina cristalina o de análogos de acción ultrarrápida, así como también las insulinas premezcladas $70 / 30^{51}$.

\section{Pramlintide: presentación e indicaciones}

Se presenta en viales de $5 \mathrm{~mL}$ que contienen $0.6 \mathrm{mg} / \mathrm{mL}$ de pramlintide. Al igual que otros polipéptidos, el pramlintide (Symlin $®$ ) debe ser inyectado en el tejido celular subcutáneo del abdomen o de los muslos. Su uso requiere jeringas de insulina U 100 (preferiblemente de $0.3 \mathrm{~mL})$. La dosis inicial en diabetes tipo 2 es de $10 \mathrm{U}(60 \mathrm{mcg})$, inmediatamente antes de cada comida principal. Según la respuesta y efectos adversos puede aumentarse la dosis hasta $20 \mathrm{U}$ (I $20 \mathrm{mcg})$. En diabetes tipo I se inicia con $2.5 \mathrm{U}$ ( $15 \mathrm{mcg})$, antes de cada comida principal. La dosis puede ser aumentada a $5 \mathrm{U}$ (30 mcg), 7.5U (45 mcg) ó IOU $(60 \mathrm{mcg})^{5 !}$.

Está indicado en pacientes con DMI ó DM2 que estén utilizando insulina cuando no se llegue a un buen control glucémico, a pesar de un manejo individualizado y óptimo de la insulinoterapia por un médico entrenado en diabetes (demostrado por una $\mathrm{HbAlc}<9.0 \%$ ), y siempre apoyado en un adecuado programa de educación ${ }^{51}$. No debe prescribirse si no se tiene un contexto adecuado para el manejo de la diabetes. Está contraindicado en casos de gastroparesia, desconocimiento de la hipoglucemia o hipersensibilidad al medicamento ${ }^{51}$.

\section{INSULINA INHALADA}

\section{Mecanismo de acción}

La insulina en todas sus presentaciones comerciales es el medicamento más destacado para el manejo de la DMI y DM2. Sus acciones superan en conjunto a cualquier otro fármaco antidiabético y apuntan a solucionar varios defectos encontrados en la diabetes, ya que resuelve íntegramente el déficit insulínico encontrado en la DMI y la insuficiencia relativa de la secreción de insulina en DM2, a través de su suplencia exógena, permitiendo de esta manera reducir tanto glucotoxicidad como lipotoxicidad (esta última al promover la captación y almacenamiento de grasas por el tejido adiposo, mediante el estímulo de la lipoproteín lipasa I y el bloqueo de la lipólisis tras la inhibición de la lipasa adipolítica $\left.{ }^{53}\right)$.

Existen varios sistemas para administrar insulina, entre los que se encuentran: los inyectores «jet», los infusores, las bombas de infusión continua subcutánea de insulina, los estilógrafos (pens de insulina) y las jeringas de insulina. Sin embargo, todos estos son métodos invasivos.

Actualmente se encuentra disponible una alternativa para administrar insulina de una manera no invasiva por vía pulmonar a través de inhalación, aprovechando las características del epitelio alveolar que expone una superficie permeable de más de 100 metros cuadrados, 10 que le proporciona un área amplia para la absorción de medicamentos $^{54}$. Cuando la insulina alcanza el espacio alveolar, atraviesa los neumocitos por transcitosis, accediendo de esta forma a la circulación ${ }^{55}$.

Entre este nuevo grupo de medicamentos encontramos Exubera ${ }^{\circledR}$, una insulina de acción rápida humana producida por ADN recombinante en forma de polvo para ser inhalada.

\section{Evidencia}

La eficacia de la terapia con insulina inhalada fue evaluada en dos meta análisis ${ }^{56,57}$ que incluyeron estudios clínicos controlados y aleatorizados en pacientes con DMI o DM2. Los estudios tuvieron una duración mínima de 12 semanas. Los meta análisis lograron concluir que:

- La insulina inhalada tiene una eficacia levemente inferior a la insulina subcutánea. Sin embargo, no hubo diferencias en la proporción de pacientes que llegaron a tener una $\mathrm{HbAlc}<7 \%$ en ambos grupos ${ }^{56}$.

- La insulina inhalada tiene una eficacia superior a los antidiabéticos orales (HbAlc - I.04\%). Esta eficacia disminuye si los antidiabéticos orales son titulados para alcanzar las metas de glucemia $(-0.20 \%)$. Sin embargo, más pacientes alcanzaron una $\mathrm{HbAlc}<7 \%$ en el grupo de insulina inhalada que en el grupo de antidiabéticos orales $(30.9 \% \text { vs } 16.9 \%)^{56}$.

- No hubo diferencias en ganancia de peso entre la insulina inhalada y la insulina subcutánea ${ }^{56,57}$. Sin embargo, los pacientes con insulina inhalada aumentaron en promedio I.85 kilos cuando se comparó con la terapia oral (pero con rosiglitazona no hubo diferencias) ${ }^{56}$.

\section{Efectos adversos}

La hipoglucemia no fue diferente entre aquellos que utilizaron insulina subcutánea comparado con quienes usaron insulina 
inhalada ${ }^{54,56,57}$. Se encontraron niveles de anticuerpos más altos en los pacientes que utilizaron insulina inhalada comparado con la insulina subcutánea, pero no parecen revestir importancia clínica ${ }^{56,57}$.

Por ser un polipéptido, la insulina puede generar una respuesta inmune en los pulmones, por tanto, los efectos adversos respiratorios son relevantes. La tos no productiva es un efecto adverso frecuente, que se presenta en alrededor del $20 \%$ de los usuarios de la insulina inhalada ${ }^{56,57}$. La tos es leve, se presenta segundos o minutos luego de la inhalación y disminuye la frecuencia a lo largo del tiempo ${ }^{56,57}$. La insulina inhalada disminuye levemente ciertas pruebas de función pulmonar (volumen espiratorio forzado al primer segundo -VEFI - y la capacidad de difusión de monóxido de carbono -DLCO-), lo que no progresó a lo largo de dos años ${ }^{56}$. Sin embargo, se necesitan estudios de más amplio seguimiento y una cuidadosa vigilancia postmarketing, para evaluar totalmente los efectos a largo plazo de la insulina inhalada sobre la función pulmonar ${ }^{56}$.

Otros efectos adversos reportados fueron disnea (aprox. 4.0\%), producción de esputo (aprox. 3.0\%), boca seca (2.4\%), epistaxis (aprox. 1.2\%), y alteraciones de la voz (aprox. 1.0\%)

\section{Presentación e indicaciones}

Exubera ${ }^{\circledR}$ es la primera insulina de acción rápida aprobada para el tratamiento de la DMI y la DM2. Se presenta en blísteres unidosis que contienen I ó 3 mg de insulina en polvo, que se administra vía pulmonar mediante inhalación oral únicamente con el inhalador de insulina ${ }^{59}$. El inhalador de Exubera ${ }^{\circledR}$ genera un pulso del aire comprimido y así desaglomera el polvo seco en un aeroso ${ }^{160}$.

En este caso, la dosis de insulina se da en miligramos, siendo I mg equivalente a 3 unidades de insulina regular subcutánea y $3 \mathrm{mg}$ equivalente a 8 unidades de insulina regular subcutánea, por lo tanto no se debe reemplazar tres blísteres de I mg por uno de $3 \mathrm{mg}^{58}$. Se sugiere una dosis inicial de $0.05 \mathrm{mg}$ por kilo de peso para ser administrada 10 minutos antes de las comidas.

Está indicado en pacientes con DMI junto con una terapia con insulinas de acción prolongada y en DM2 como monoterapia o en combinación con antidiabéticos orales, o insulinas de acción prolongada ${ }^{58}$.

Su uso está contraindicado en personas que han fumado durante los últimos 6 meses (ya que el tabaquismo aumenta la absorción del medicamento de dos a cinco veces por mecanismos aún no dilucidados $\left.{ }^{6}\right)$, así como también en personas con enfermedad pulmonar inestable o pobremente controlada ${ }^{58}$.

Los pacientes que inician esta alternativa insulínica deberían tener previamente una espirometría y controlar la función pulmonar seis meses después, y luego cada año aún si no se refieren síntomas. Si se presenta una disminución del VEF $1 \geq 20 \%$ la terapia debería suspenderse ${ }^{58}$.

Sin embargo, en octubre de 2007 la empresa productora de Exubera anunció que este fármaco saldría del mercado por sus bajas ventas. El medicamento estuvo disponible hasta enero de $2008^{62}$.

La mayor limitante de la gran mayoría de estas novedosas terapias para la diabetes es su elevado costo, frente a otras alternativas farmacológicas previas más asequibles. De igual forma, estas terapias no son superiores a los medicamentos tradicionales puesto que son tan eficaces como algunos fármacos ya conocidos (como metformina o insulina inyectable). Es de resaltar el hecho de que se abren alternativas para la terapia de la DMI, que hasta hace poco tiempo sólo contaba con insulina parenteral y hoy se puede considerar la terapia con agonistas de la amilina.

En conclusión, el abanico terapéutico para la diabetes ha aumentado y seguirá aumentando en el futuro con la llegada de nuevas familias farmacológicas, como los inhibidores de los cotransportadores de sodio y glucosa 2 (SGLT2), que impiden la reabsorción renal de glucosa permitiendo su excreción urinaria con la consecuente reducción en los niveles de glucemia ${ }^{63}$ o la modulación de ciertos receptores de ácidos grasos acoplados a proteína $\mathrm{G}$ involucrados en la secreción de insuli$n a^{64}$, entre otros medicamentos.

\section{SUMMARY}

\section{New therapies fOR diabetes: beYond INJeCtAble INSULIN AND ORAL ANTIDIABETICS}

New medicines for the therapy of the type I and type 2 diabetes have been incorporated in the list of traditional drugs: oral agents and injectable insulin. These treatmentalternatives have a new mechanism of action that takes advantage of the antidiabetic properties of certain peptides such as amylin and glucagon like peptide-I (GLP-I), whose levels are wanting or insufficient in diabetes. This is attained through amylin and GLP-I analogues, although it can also be achieved by inhibiting the enzyme that degrades the latter. Furthermore, a new system to administer insulin in a noninvasive way through inhalation has become available in the market. This paper summarizes the most important and updated findings on the action mechanism, efficacy, adverse effects and indications of these innovative drugs. [Rev Assoc Med Bras 2008; 54(5): 447-54]

KEY wORDS: Hypoglycemic agents. Diabetes mellitus type I. Diabetes mellitus type 2. Glucagon-like peptide I. Dipeptidyl peptidase IV. Insulin.

\section{Bibliografía}

I. Guías ALAD 2006 de diagnóstico, control y tratamiento de la diabetes mellitus tipo 2. Bogota: Asociación Latinoamericana de Diabetes; 2006.

2. Nauck MA, Homberger E, Siegel EG, Allen RC, Eaton RP, Ebert R, et al. Incretin effects of increasing glucose loads in man calculated from venous insulin and C- peptide responses. J Clin Endocrinol Metab. 1986;63:492-8.

3. Ahrén B. Dipeptidyl peptidase-4 inhibitors. Diabetes Care. 2007;30: 1344-50.

4. Drucker DJ, Nauck MA. The incretin system: glucagon-like peptide-I receptor agonists and dipeptidyl peptidase-4 inhibitors in type 2 diabetes. Lancet. 2006;368: I 696-705.

5. Schirra J, Nicolaus M, Roggel R, Katschinski M, Storr M, Woerle HJ, et al. Endogenous GLP-I controls endocrine pancreatic secretion and antropyloro-duodenal motility in humans. Gut. 2006;55:243-51 .

6. Perfetti R, Zhou J, Doyle M, Egan JM. Glucagon like peptide-I induces cell proliferation and pancreatic-duodenum homeobox-I expression and increase cell mass in the pancreas of old glucose intolerant rats. Endocrinology. 2000; 1 41:4600-5.

7. Farilla L, Bulotta A, Hirshberg B, Li Calzi S, Khoury N, Noushmehr H. Glucagon-like peptide I inhibits cell apoptosis and improves glucose 
responsiveness of freshly isolated human islets Endocrinology. 2003; | 44:5 | 49-58.

8. Meier JJ, Gallwitz B, Salmen S, Goetze O, Holst JJ, Schmidt WE, et a.I Normalization of glucose concentrations and deceleration of gastric emptying after solid meals during intravenous glucagon-like peptide I in patients with type 2 diabetes.J Clin Endocrinol Metab. 2003;88:27 I 9-25.

9. Turton MD, O'shea D, Gunn I, Beak SA, Edwards CM, Meeran K, et al. A role for glucagon peptide-I in the central regulation of feeding. Nature. 1996:379:69-72

10. Eng J, Kleinman WA, Singh L, Singh G, Raufman JP. Isolation and characterization of exendin-4, an exendin-3 analogue, from Heloderma suspectum venom. Further evidence for an exendin receptor on disprsed acini from guinea pig pancreas. J Biol Chem. 1992;267:7402-5.

II. Buse JB, Henry RR, Han J, Kim DD, Fineman MS, Baron AD. Effects of exenatine (exendin-4) on glicemic control over 30 weeks in sulfonylureatreated patients with type 2 diabetes. Diabetes Care. 2004;27:2628-35.

12. De Fronzo RA, Ratner RE, Han J, Kim DD, Fineman MS, Baron AD. Effects of exenatide (exendin-4) on glycemic control and weight over 30 weeks in metformin-treated patients with type 2 diabetes. Diabetes Care. 2005:28: 1092-100.

13. Kendall DM, Riddle MC, Rosenstock J, Zhuang D, KimDD, Fineman MS. Effects of exenatide (exendin-4) on glycemic control over 30 weeks in patients with type 2 diabetes treated with metformin and a sulfonylurea. Diabetes Care. 2005;28: | 083-9|

14. Zinman B, HoogwerfBJ, Duran S, Milton DR, Giaconia G, Kim DD, etal. The effect of adding exenatide to thiazolidinedione in suboptimally controlled type 2 diabetes: a randomized trial. Ann Intern Med. 2007; I 46:477-85.

15. Heine RJ, Van Gaal LF, Johns D, Mihm MJ, Widel MH, Brodows RG. Exenatide versus insulin glargine in patients with suboptimally controlled type 2 diabetes: a ramdorized trial. Ann Intern Med. 2005; | 43:559-69.

16. Nauck MA, Duran S, Kim D, Johns D, Northrup J, Festa A, et al. A comparison of twice daily exenatide and biphasic insulin aspartat in patients with type 2 diabetes who were suboptimally controlled with sulfonylurea and metformin: a non inferiority study. Diabetologia. 2007:50:259-67

17. Kolterman OG, Kim DD, Shen L, et al. Pharmacokinetics, pharmacodynamics, and safety of exenatide in patients with type 2 diabetes mellitus. Am Health Syst Pharm 2005; 62: 173-181

18. Keating GM. Exenatide. Drugs 2005; 65: 1681-1692

19. Amylin pharmaceuticals, Inc. Byetta $T$ (exenatide injection: prescribing information) [cited 2007 ago 24]. Available from: http:// www.byetta.com.

20. Kim D, MacConell L, Zhuang D, Kothare PA, Trautmann M, Fineman M, et al. Effect of once-weekly dosing of a long-acing release formulation of exenatide on glucose control and body weight in subjects with type 2 diabetes. Diabetes Care. 2007;30: | 487-93.

21. Deacon CF. Therapeutic strategies based upon glucagon-like peptide I. Diabetes. 2004:53:2 I 8I-9.

22. Vilsboll T, Zdravkovic M, Le-Thi T, Krarup T, Schmitz O, Courreges JP, et al. Liraglutide, a long-acting human glucagon-like peptide-I analog, given as monotherapy significantly improves glycemic control and lowers body weight without risk of hypoglycemia in patients with type 2 diabetes. Diabetes Care. 2007:30: 1608-10.

23. Madsbad S, Schmitz O, Ranstam J, Jakobsen G, Matthews DR. Improved glycemic control with no weight increase in patients with type 2 diabetes after once-daily treatment with the long-acting glucagon-like peptide analog lirtaglutide (NN22 I I): a 12 week, double blind, randomized controlled trial. Diabetes Care. 2004;27: 1335-42.

24. Feinglos MN, Saad MF, Pi Sunyer FX, An B, Santiago O. Effects of liraglutide (NN22 I I), a long-acting GLP-I nalogue, on glycaemic control and bodyweight in subjects with type 2 diabetes. Diabetes Med 2005;22: 1 016-23

25. FierceBiotech. Press release: liraglutide improves glucose control and lowers body weight in two phase 3 studies comprising [cited 2007 aug 24]. Disponible en URL http://www.fiercebiotech.com/press-releases/ press-release-liraglutide-improves-glucose-control-and-lowers-bodyweight-two-phase-3. [Consultado 24 de agosto de 2007]
26. Amori RE, Lau J, Pittas AG. Efficacy and safety of incretin therapy in type 2 diabetes: systematic review and a meta-analisis. JAMA 2007; 298: 194-206

27. Johl CB, Hollingdal M, Sturis J, Jakobsen G, Agerso H, Veldhuis J, et al. Bedtime administration of NN22I I, a long-acting GLP-I derivative, substancially reduces fasting and postprandial glycemia in type 2 diabetes. Diabetes 2002; 51: 424-429

28. Ahren B, Landin-Olsson M, Jansson PA, Svensson M, Holmes D, Schweizer A: Inhibition of dipeptidyl peptidase-4 reduces glycemia, sustains insulin levels and reduces glucagon levels in type 2 diabetes. J Clin Endocrinol Metab 2004; 89: 2078-2084

29. Mari A, Sallas WM, He L, Watson C, Ligueros-seylan M, Dunning BE, et al. Vildagliptin, a dipeptidyl peptidase-IV inhibitor improves modelassessed beta cell function in patients with type 2 diabetes. J Clin Endocrinol Metab 2005; 90: 4888-4894

30. Nauck MA, Mininger G, Sheng D, Terranella L, Stein PP. Efficacy and safety of the dipeptidyl peptidase-4 inhibitor, sitagliptin, compared with the sulfonlurea, glipizide, in patients with type 2 diabetes inadecuately controlled on metformina alone: a randomized, double-blind, noninferiority trial. Diabetes Obes Metab 2007; 9: 194-205

31. Goldstein BJ, Feinglos MN, Lunceford JK, Johnson J, Williams-Herman DE. Effect of initial combination therapy with sitagliptin, a dipeptidyl peptidase-4 inhibitor, and metformin on glycemic control in patients with type 2 diabetes. Diabetes Care 2007; 30: 1979- 1987

32. Merck \& Co, Inc. Januvia T (sitagliptin: prescribing information) [Internet]. Disponible en URL http://www.januvia.com [Consultado 28 de agosto de 2007]

33. Merck \& Co, Inc. Janumet T (sitagliptin / metformin HCL: prescribing information) [Internet]. Disponible en URL http://www.janumet.com [Consultado 28 de agosto de 2007]

34. Rosenstock J, Baron MA, Camisasca RP, Ctressier F, Couturier A, Dejager S. Efficacy and tolerabilyty of initial combination therapy with vildagliptina and pioglitazone compared monotherapy in patients with type 2 diabetes. Diabetes Obes Metab 2007; I75- 185

35. Rosenstock J, Baron MA, Dejager S, Mills D, Schweizer A. Comparison of vildagliptin and rosiglitazone monotherapy in patients with type 2 diabetes: a 24-week, doiuble-blind, randomized trial. Diabetes Care 2007: 30: 217-223

36. Schweizer A, Couturier A, Foley JE, Dejager S. Comparison between vildagliptin and metformin to sustain reductions in $\mathrm{HbAlc}$ over a year in drug naïve patients with type 2 diabetes. Diabetes Med 2007: doi: | 0.1 | | |/j.|464-549|.2007.02191.x.

37. Schlesselman LS. Vildagliptin. Formulary 2006; 4I: 494-500

38. Novartis Farmacéutica. Galvus ${ }^{\circledR}$ (Vildagliptina) Monografía del producto. México 2007

39. Mendivil CO, Sierra ID. Nuevos medicamentos antidiabéticos. En: Sierra ID, Mendivil CO, et al. Hacia el manejo práctico de la diabetes mellitus tipo 2. 3Ed. Editorial kimpres Ltda. 2006

40. Riddle MC, Drucker DJ. Emerging therapies mimicking the effects of amylin and glucagon-like peptide I. Diabetes Care 2006; 29: 435-449

41. Kruger DF, Aronoff SL, Edelman SV. Through the Looking Glass: Current and Future Perspectives on the Role of Hormonal Interplay in Glucose Homeostasis. Diabetes Educ 2007; 33(Suppl2): 32S - 46S.

42. Ryan GJ, Jobe LJ, Martin R. Pramlintide in the treatment of type I and type 2 diabetes mellitus. Clin Therapeutics 2005; 27: I 500- I 5 I2.

43. Buse JB, Weyer C, Maggs DG. Amylin replacement with pramlintide in type I and type 2 diabetes: A physiological approach to overcome barriers with insulin therapy. Clin Diabetes. 2002;20: 137-I44.

44. Lutz TA, MolletA, Rushing PA, et al. The anorectic effect of a chronic peripheral infusion of amylin is abolished in area postrema/nucleus of solitary tract (AP/NTS) lesioned rats. Int J Obes Relat Metab Disord. 200 I; 25:1005-1011.

45. Chapman I, Parker B, Doran S, Feinle-Bisset C, Wishart J, Strobel S, et al. Effect on pramlintide on saciety and food intake in obese subjects and subjects with type 2 diabetes. Diabetologia 2005; 48: 838-848

46. Koda JE, Fineman M, Rink TJ, Dailey GE, Muchmore DB, Linarelli LG. Amylin concentrations and glucose control. Lancet 1992; 339:1179. II80. 
47. Ludvik B, Lell B, Hartter E, Schnack C, Prager R. Decrease of stimulated amylin release precedes impairment of insulin secretion in type 2 diabetes. Diabetes 1991; 40:1615-1619.

48. Ratner RE, Want LL, Fineman MS, Velte MJ, Ruggles JA, Gottlieb A, et al. Adjunctive Therapy with the Amylin Analogue Pramlintide Leads to a Combined Improvement in Glycemic and Weight Control in InsulinTreated Subjects with Type 2 Diabetes. Diabetes Technol Ther 2002 ; 4: 5|-6|.

49. Hollander PA, Levi P, Fineman MS, Maggs DG, Shen LZ, strobel SA, et al. Pramlintide as an adjunct to insulin therapy improves long-term glycemic and weight control in patients with type 2 diabetes: a I-year randomized controlled trial. Diabetes Care. 2003; $26: 784-790$.

50. Maggs D, Shen L, Strobel S, Brown D, Kolterman O, Weyer C. Effect of pramlintide on AIC and body weight in insulin-treated African Americans and Hispanics with type 2 diabetes: a pooled post hoc analysis. Metabolism. 2003; 52: 1638-1642.

51. Amylin pharmaceuticals, Inc. Symlin T (pramlintide acetate injection: prescribing information) [Internet]. Disponible en URL http:// www.symlin.com [Consultado 3 de septiembre de 2007]

52. Singh-Franco D, Robles G, Gazze D. Pramlintide acetate injection for the treatment of type I and type 2 diabetes mellitus. Clin Therapeutics 2007; 29: $535-562$

53. Mendivil CO, Sierra ID. Acción insulínica y Resistencia a la insulina: aspectos moleculares. Rev Fac Med Univ Nac Colomb 2005; 53: $843-$ 853.

54. Hollander PA. Evolution of pulmonary insulin delivery system (Exubera) for patients with diabetes. MedGenMed 2007; 9: 45

55. Mendivil CO, Sierra ID. Insulinoterapia. En: Sierra ID, Mendivil CO, et al. Hacia el manejo práctico de la diabetes mellitus tipo 2. 3Ed. Editorial kimpres Ltda. 2006
56. Ceglia L, Lau J, Pittas AG. Meta-analysis: Efficacy and safety of inhalated insulin therapy in adults with diabetes mellitus. Ann Intern Med 2006; I 45: 665-675

57. Royle P, Waugh N, McAuley L, McIntyre L, Thomas S. Inhaled insulin in diabetes mellitus. Cochrane Database of Systematic Reviews 2003, Issue 4. Art. No.: CD003890. DOI: I 0. I 002/I 465 | 858.CD003890.pub2.

58. Pfizer Inc. ExuberaT (insulin human [rDNA origin]) Inhalation Powder: prescribing information) [Internet]. Disponible en URL http:// www.exubera.com [Consultado 7 de septiembre de 2007]

59. Pfizer Inc. ExuberaT (insulina humana inhalada: Ficha técnica) [Internet]. Disponible en URL http://www.pfizer.es/FichasTecnicas/FTEXUBERA\% 203MG-MAR07-V04.pdf [Consultado 7 de septiembre de 2007]

60. Ghosh S, Collier S. Inhaled insulins. Postgrad Med J 2007; 83: |78-8|

61. Becker RH, Sha S, Frick AD, Fountaine RJ. The effect of smoking cessation and subsequent resumption on absortion of inhaled insulin. Diabetes Care 2006; 29: 277-282

62. Pfizer Inc. ExuberaT [Internet]. Disponible en: http://www.exubera.com/ content/con index.jsp?setShowOn=../content/con index.jsp\&set ShowHighlightOn=../content/con_index.jsp [Consultado II de Enero de 2008]

63. Isaji M. Sodium-glucose cotransporters inhibitors for diabetes. Curr Opin Investig Drugs 2007; 8: 285-292

64. Rayasam GV, Tulasi VK, Davis JA, Bansai VS. Fatty acid receptors as new therapeutic targets for diabetes. Expert Opin Ther 2007; I I: 66 I-67|

Artigo recebido: | 4/0|/08

Aceito para publicação: 14/03/08 